\title{
Research on Intelligent Shelf based on Raspberry Pi Software and Big Data
}

\author{
Shugang Liu ${ }^{a,}{ }^{*}$, Mingchun Wang ${ }^{\mathrm{b}}$ and Manna Liu ${ }^{\mathrm{c}}$ \\ Department of Computer Science and Engineering, North China Electric Power University, \\ Baoding 071000, China \\ a, ${ }^{*}$ 274786113@qq.com, b2542921704@qq.com, c2310278267@qq.com
}

\begin{abstract}
With the development of science and technology, intelligent facility has already become an indispensable part of our life. At the same time, shopkeepers want a convenient and inexpensive facility to cut the labor cost. To meet their needs, we decide to design an Intelligent Shelf System. The Intelligent Shelf System should include four parts: shelf management, behavior identification, data upload and image update. After the system has been tested, we find that the system can identify $98.4 \%$ of goods on average.
\end{abstract}

Keywords: Raspberry Pi; The Intelligent Shelf System; Image Processing.

\section{Introduction}

\subsection{Background}

In order to have better understanding of our intelligent shelf, the following background is worth mentioning.

In recent years, with rapid increase of labor cost, shopkeepers spend more money in hiring people and make a less profit. It has a more obvious impact on small retail stores than large-sized chain supermarkets since small retail stores are lack of capital and easier to go bankrupt in this circumstance. The other risk that shopkeepers of retail stores have to face is the wrong behaviors of employees and customers such as shoplifting. Shoplifting and mistakes made by employees always lead to missing of goods and result in financial loss. While trying hard to solve the problem, people find that it is almost impossible for employees to concentrate on their work for a long time. In this aspect, computers may be a better choice to do such an energy-consuming work. During a long period of investigation on the local retail stores, our team decides to design an Intelligent Shelf System based on the use of Raspberry Pi. We believe that our product can cut the labor cost and provide people with more convenient service.

\subsection{Problem Analysis}

By analyzing the needs of shopkeepers of retail stores, we know that an Intelligent Shelf System must have the function to provide, save, update and search data of different goods. We conclude the following information:

(a)After logging in the system, shopkeepers can not only maintain data of shelves and goods, but monitor everything that has happened in the stores, such as the positions of goods and what customers have done.

(b)Every shelf and every good has its own unique code to locate its' position.

(c)Raspberry Pi can identify what kind of behavior that customers have done by analyzing and calculating the positional changes of goods.

(d)Results are uploaded to the server side, the shopkeeper's personal computer, as long as raspberry pi finishes its calculation.

\subsection{Objectives}

According to what has been mentioned above, the design of the Intelligent Shelf System includes four parts: shelf management, behavior identification, data upload and image update. A complete procedure is as followed: 
First, customers can open the door by scanning the QR code on the shelf. At the same time, Raspberry $\mathrm{Pi}$, which is fixed on the inner side of the shelf, takes a photo automatically. After closing the door, Raspberry Pi takes another photo. Then it compares the two photos and draws the conclusions. At last, the conclusions will be sent to the server side.

To demonstrate better how the Intelligent Shelf System works, we use two flow charts showing the operation flow and functional module respectively in Figure 1 and Figure 2.

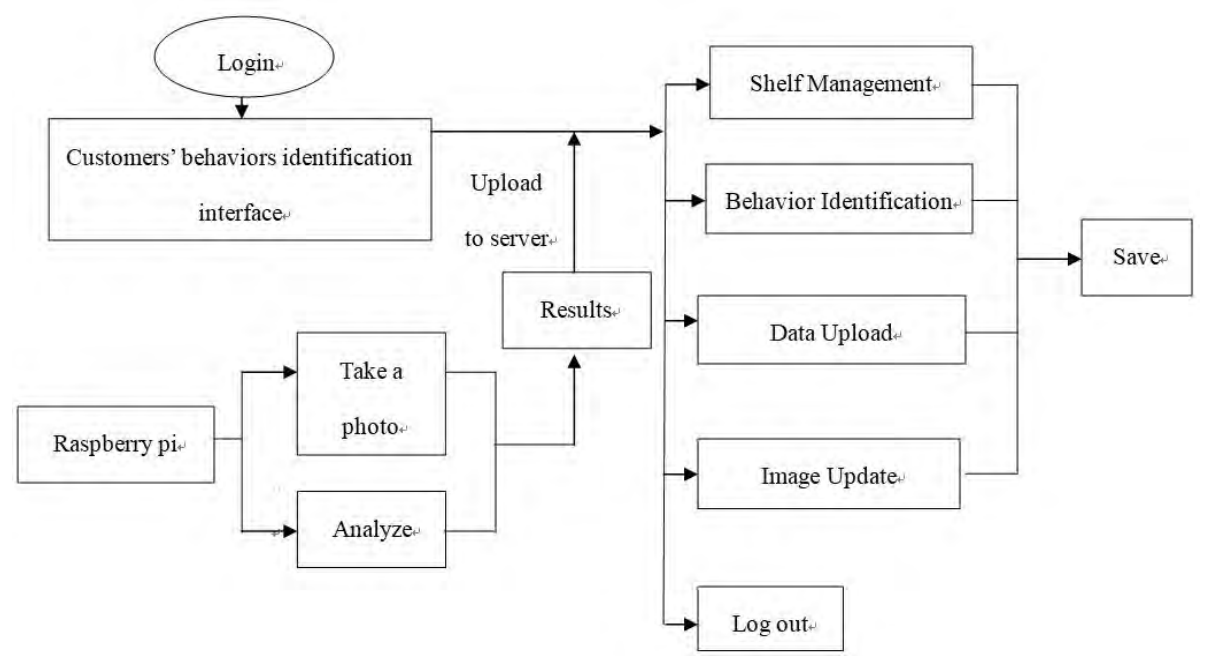

Figure 1. The flow chart which shows the operational flow.

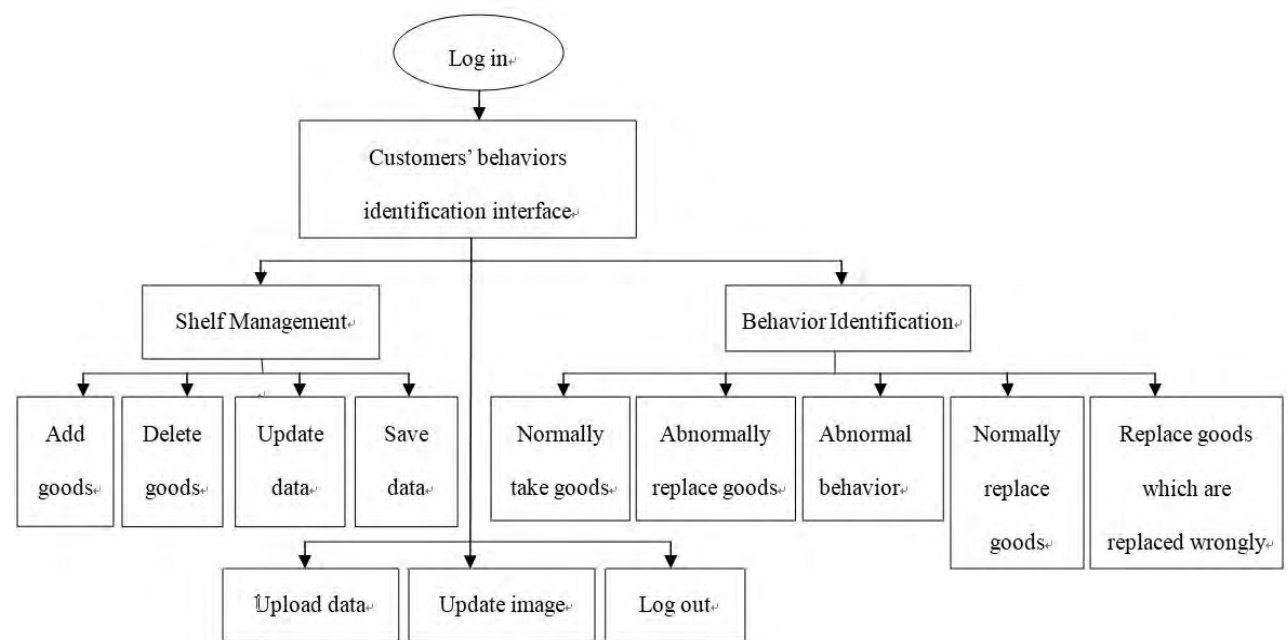

Figure 2. The flow chart which shows the functional module.

\section{Main Procedures}

We divide the procedures into four parts to implement our functions: install Raspberry Pi, design Behavior Identification System, and build connection between the client side and the server side.

\subsection{Install Raspberry Pi}

First, we should write operation system into SD card of Raspberry Pi before turn on it. Second, we link Raspberry Pi to router. Since our computer and Raspberry Pi are on the same WIFI network, we can easily find IP address of Raspberry Pi and have remote access to Raspberry Pi by using Putty and VNC Viewer. In fact, Raspberry Pi is a minicomputer in the Intelligent Shelf System.

\subsection{Design Behavior Identification System}

We use python to write our application program in Raspberry Pi. Considering that parameters such as shape and position vary from shelf to shelf, we design a demonstration system to help shopkeepers 
set parameters of goods. Moreover, we can check if the results are correct with the demonstration system.

\subsection{Build Connection}

Raspberry Pi acts as the client side while personal computer acts as the server side. After exporting photos to personal computer and setting their parameters, we execute Shelfsys.exe in personal computer and picamNEW.py in Raspberry $\mathrm{Pi}$ in order to link up the client side and the server side. Shelfsys.exe is a program showing results sent by Raspberry Pi and picamNEW.py is the application program we have mentioned above.

\section{Result Presentation}

\subsection{Structure of the Shelf}

Figure 3 shows the front of the shelf. The top of the shelf is a computer screen which displays the result of analysis. We use electrical relay to send the control signal of opening or closing door. To improve photographic quality, each layer of the shelf is equipped with flash lights.

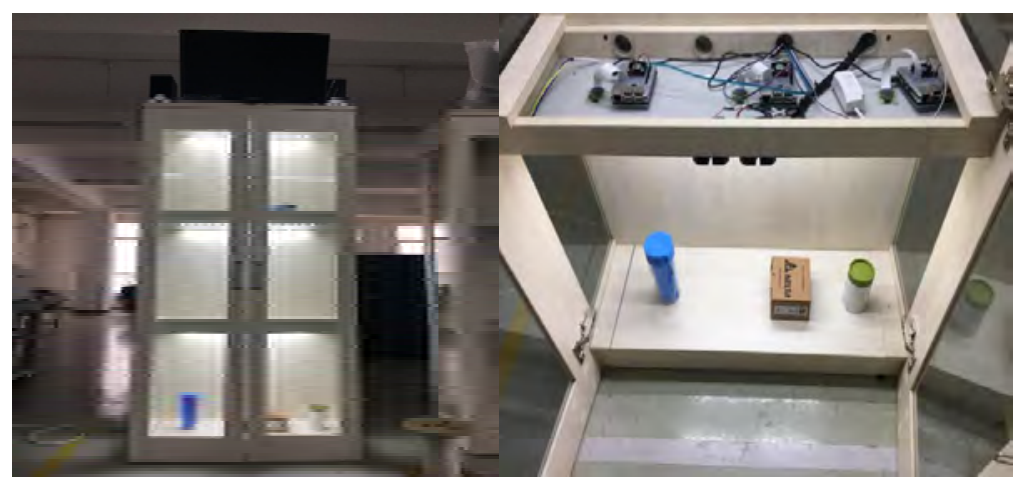

Figure 3. To show the front of the shelf.

\subsection{Installation Procedures of Raspberry Pi}

Figure 4 shows that Raspberry Pi is fixed on the inner side of the shelf. We use it to calculate and analyze data. There are some small holes on the layers to make photograph more convenient.

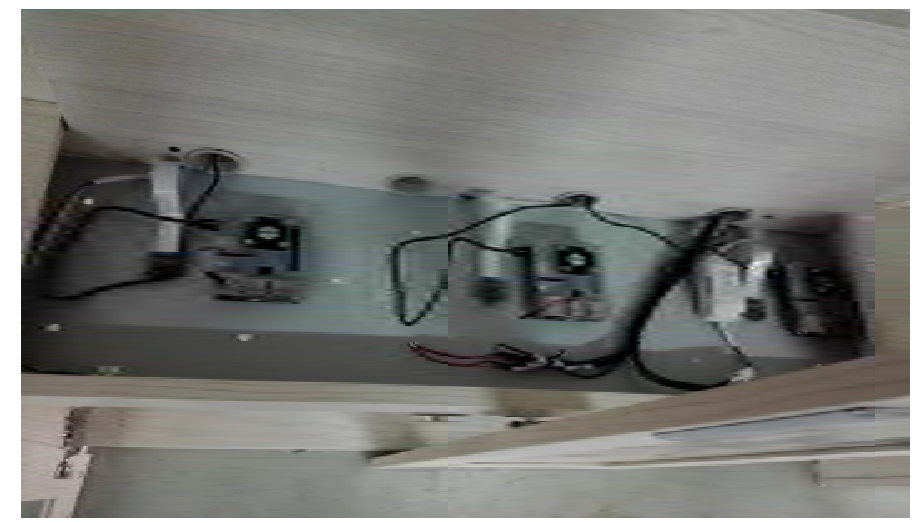

Figure 4. To show the inner side of the shelf.

\subsection{Remote Access to Raspberry Pi}

Figure 5 shows the desktop window of Raspberry Pi. Raspberry Pi acts as a mini computer. Our computers can have remote access to Raspberry Pi by using Putty and VNC Viewer. 


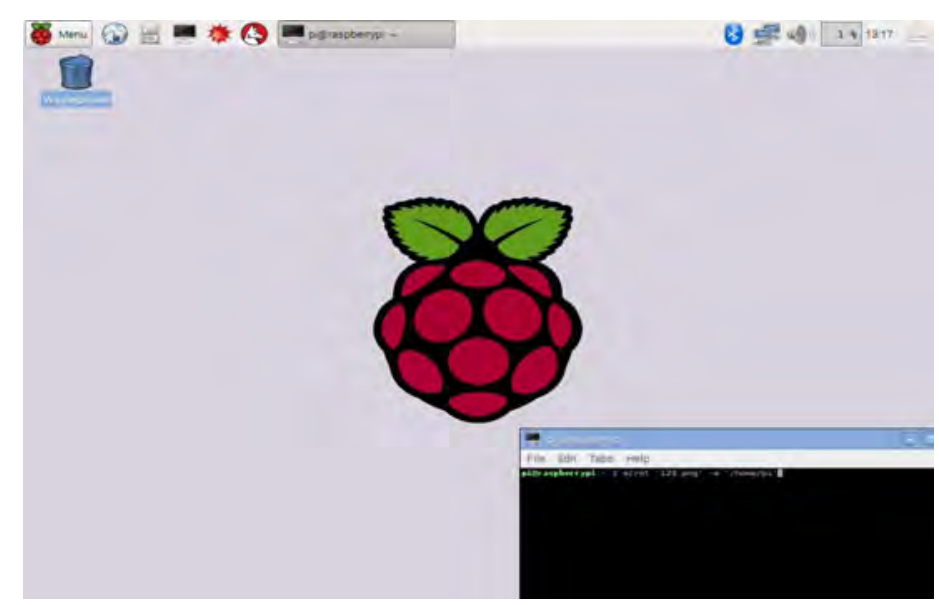

Figure 5. To show the desktop window of Raspberry Pi.

\section{Conclusion}

Throughout many tests, we already know how well the Intelligent Shelf System worked for shelf management and behavior identification. Compared with other similar products in the market, the Intelligent Shelf System needs less data to produce results. Hence it can not only occupy less device space, but also calculate more quickly and correctly.

\section{References}

[1]. Wesley Chun, Core Python Applications Programming (Posts and Telecom Press, Peking, 2016), pp. 61-79.

[2]. Prateek Joshi, Artificial Intelligence with Python (Southeast University Press, Nanjing, 2017), pp.72-82.

[3]. Dogan Ibrahim, RASPBERRY PI: Hardware Projects (Southeast University Press, Nanjing, 2015). 